\title{
COROT and the late stages of stellar evolution
}

\author{
T. Lebzelter, H. Pikall, F. Kerschbaum \\ Institut für Astronomie, Türkenschanzstrasse 17, A-1180 Vienna, Austria
}

\begin{abstract}
Small amplitude variability is observed during the late stages of stellar evolution of low- to intermediate-mass stars. In this paper we discuss the occurrence of small-amplitude variations in the upper right part of the HRD, namely among the AGB and post-AGB stars, and we propose two observing programs for the COROT Additional Program.
\end{abstract}

\section{AGB and post-AGB evolution}

After exhaustion of central hydrogen, stars of low to intermediate mass evolve into red giants. The ascent on the first giant branch (RGB) is followed by a phase of helium core burning and a phase of helium and hydrogen shell burning, the latter being called the Asymptotic Giant Branch phase (AGB). This phase is characterized by cool, highly extended stellar atmospheres/envelopes. Pulsation is a critical aspect of the late stages of stellar evolution. Regular, semiregular and irregular variations have been found in the light and velocity changes of these stars. Based on regularity, period and amplitude three types of variables have been defined: Miras, Semiregular variables (SRVs) and Irregular variables (Lb). Pulsation affects the structure of the stellar atmosphere. Expansion leads to conditions better suited for the formation of dust. Mass loss and dust production are related and important tests for stellar evolution While variability is probably mainly due to pulsation, it may be affected by other sources. The attempt to understand the irregularities in the light change of these stars is one of the main aspects currently investigated in these objects (Percy 1997, Lebzelter et al. 2000, Kerschbaum et al. 2001, Percy et al. 2001).

The AGB phase terminates when the star reaches a very high mass loss rate. While losing the remainder of its atmosphere, the degenerated core is uncovered and the effective temperature rises shifting the objects to the "left" in the HRD. 
When all nuclear sources of energy are used up, the final (core-)contraction is inevitable and the objects arrive at the white dwarf (WD) cooling track, which is characterized by decreasing luminosity and temperature. A number of interesting phases are associated with these final steps in stellar evolution. Here we want to focus on three classes of objects: R CrB stars (RCB), Hydrogen deficient Carbon-stars ( $\mathrm{HdC}$ ) and Extreme Helium Stars (eHe). All three groups are characterized by the absence of Hydrogen. While for the first two groups $\mathrm{H}$ is underabundant by a factor of $10^{-4}$ to $10^{-8}$ (number density), some objects among the eHes have a larger fraction of $\mathrm{H}$.

Following Iben et al. (1996) two evolutionary scenarios are thought to lead to the $\mathrm{R} C r B$ phenomenon: One possibility is the merging of two, close degenerated objects - like WD or even Neutron stars - in a binary system (double degenerate scenario, DD). Recent calculations of WD-evolution with He-accretion (Saio \& Jeffery 2001) support this idea. The accreted material is heated and compressed by shocks which starts the nuclear fusion. He Shell burning releases enough luminosity to virtually move the star to the upper right corner of the HRD. Alternatively a final Helium flash can produce enough luminosity to expand the envelope so the object appears as a cool supergiant again. Helium flashes occur regularly during the final stages of AGB evolution, but in some cases a very late FINAL flash may happen after the star has lost its envelope and is already on the WD cooling track (final flash scenario, FF). Both scenarios lead again to a cool, extended envelope, so that these stars are found close to the AGB.

According to Clayton (1996) all RCB have an infrared excess, while for none of the $\mathrm{HdC}$ was an infrared excess observed. IRAS data imply dust temperatures in the range of 650-900 K (Walker 1986). There is evidence for old, fossil dust shells with large spatial dimensions, implying an age of $10^{4}$ to $10^{5}$ years for the shell around R CrB itself (Gillett et al. 1986), but without a connection to the recent mass loss, which is responsible for the declines. The emisson in the infrared is changing but not correlated to the big declines. Measurements of the polarisation support the existence of dust.

Beside the obvious large amplitude variations in RCB, the declines, which are due to phases of heavy mass loss and the emission of dust in puffs directly in the line of sight, small amplitude pulsation is observed in these stars. However, investigation of these variations has just begun and their origin and type of pulsation is not yet known. Typical pulsation periods are 30-110 days for RCB and $\mathrm{HdC}$ and between 0.1 and a few days for the eHe (Weiss et al. 1996, and references therein). Feast (1996) points out that the turnover timescales for a few large convection cells are of the same order for cool objects, but there is doubt if the change in brightness can be explained by these. 


\title{
Open questions
}

\author{
AGB stars
}

Our interest in COROT concerning AGB stars is motivated by two open questions that require precise photometry with a high time resolution. At first glance, neither requirement seems necessary for investigations of AGB stars, as the variations in these objects typically occur on a time scale of several tens of days up to several years with amplitudes between 0.1 and 8 magnitudes.

However, the definition of the variability region in the HRD, where the AGB stars are located, can be established by much smaller variations on much smaller timescales. AGB variables are not located within the classical instability strip but seem to form a separate instability region. The blue border of this region is not known yet. Several investigations detected small amplitude variability in late K- and early M-type giants, i.e. on the left side of the AGB. Jorissen et al. (1997) and Fekel et al. (2000) showed that small amplitude variability is common in this kind of stars. Semiregular and irregular variables with amplitudes of more than $0.1 \mathrm{mag}$ are found also among early M-type giants (e.g. Lebzelter 1999a). Edmonds \& Gilliland (1996) found variable K-giants in the globular cluster 47 Tuc with period lengths of a few days and amplitudes of about $10 \mathrm{mmag}$. Koen \& Laney (2000) detected short time variations (periods of a few days) in $M$ giants, although part of their results may be due to problems with the Hipparcos data they used (Kerschbaum et al. 2000). A more detailed investigation of the variability in this region of the HRD is necessary to derive the origin of the observed variability and to deduce the parameters for the onset of pulsation among cool giants.

Also for the classical, large amplitude AGB variables, photometry with high time resolution is needed. It is suspected that these stars show also variations that do not origin from stellar pulsations. Opposite to what is found in Sun-like stars, the surface of a cool AGB giant should be covered only by a few large convective cells (Schwarzschild 1975; Freytag et al. 1997). Their occurrence and motion may be echoed in small amplitude changes of the star (e.g. Lebzelter 1999b). Beside that several investigators report the observation of short time outbursts in MIRAS (Maffei \& Tosti 1995, de Laverny et al. 1998). For a few hours the star's brightness changes by up to several 0.1 mag. The mechanism of these outbursts is not understood yet.

Both kinds of variations, due to surface structures and due to outbursts, happen on a short time scale and may be detectable only with high precision photometry. Due to the lack of appropriate models an accurate prediction of the time scales and amplitudes of these variations cannot be made. 


\section{Post-AGB stars}

The small amplitudes of the pulsation detected or expected in the groups of post-AGB stars described above should predestine these objects for high precision continuous photometry offered by COROT. Periods have been derived only for a small fraction (2) of the known objects, all with rather big uncertainties, so there is a clear demand for a detailed study of these stars.

The determination of exact stellar parameters like effective temperature, luminosity, mass and the chemical composition are needed to calculate detailed models of these objects - both for evolutionary and pulsational aspects. The Hipparcos mission could not determine distances to any of the objects, because many of the objects were not bright enough, but the detection of RCB in the LMC during the MACHO-project (Alcock et al. 1996) supported high luminosities. Estimates of the brightness were $M_{\mathrm{V}} \approx-4$ to -5 . This however doesn't constrain evolutionary models (which could shed light on the object's masses) sufficently. Atmospheric abundances, derived from detailed static modeling of the atmospheres, simultaneously yield values for temperatures and surface gravities (Asplund et al. 2000, Pandey et al. 2001). Pulsation theory relates the period of pulsation to the star's mean density regarding the pulsation mode, which makes it important to measure the period with high accuracy, but periods of the order of 30-110 days are hard to observe, due to seasonal effects. The temporal change of period is an indication if the star is contracting or expanding, but prior to that the period has to be established.

High luminosity in stellar envelopes allows strange modes, which can grow on very short timescales, comparable to a few of their pulsation periods. Making it simple, one could say, that such envelopes tend to show instabilities of greater order and manifold than low-luminosity objects. The reason derives partly from the fact that the Kelvin-Helmholtz timescale becomes comparable to the dynamical timescale for very high luminosities. Linear theory showed that a density inversion enables mode trapping in the outer parts of the envelopes [REFERENCE?]. Stellar winds are thought to be driven by different mechanisms, but high luminosities help anyway, if the matter is not heated, but accelerated. The connection between pulsation, wind and dust formation is still not understood. While dust formation and dust driven winds can be modeled simultaneously (Höfner 1999), the influence on the pulsation has not been investigated so far. This also leads to the question if all RCB are pulsating, and what the correlation of systematic period variations to the evolution of these objects is.

Another important question is: How does convection work in cool supergiants? As for AGB stars, which are in many ways similar to the RCB and $\mathrm{HdC}$, short time variations with low amplitudes are expected. In the case of some eHe stars the question arises: Is the observed irregularity intrinsic or due 
to shortcomings in the observations.

\section{Perspectives}

Within the course of the Additional Program we propose two observing programs. M giants and AGB stars are found all over the sky so that we aim to measure the light variability of both kinds of stars that are in the final fields of view of COROT. The data will contribute to both questions discussed for the AGB stars above. We plan to combine these observations with spectroscopic measurements and photometric follow up studies from the ground.

The second observing proposal aims to measure the light change of at least one post-AGB object with high photometric precision. It turned out that a number of stars from the three groups described in this paper would be close to the planned field of view of COROT. One star, FH Sct - an RCB - will be in the Exoplanetary Field if one of the prime targets is observed in the asteroseismology field. No pulsational period has been derived for this star yet. The apparent brightness is $V=12.2$ mag and therefore FH Sct fits perfectly in the sensitivity range of the instrument. For both proposals the Exoplanetary Field offers sufficient time resolution.

Acknowledgments. TL is supported by the Austrian Science Fund Project P14365-PHY. We wish to thank W.W.Weiss for organizing the COROT Science Week in Vienna.

\section{References}

Alcock, C., Allsman, R. A., Alves, D. R., et al. 1996, ApJ 470, 583

Asplund, M., Gustafsson, B., Lambert, D. L., Rao, N. K. 2000, A\&A353, 287

Clayton, G. C. 1996, PASP 108, 225

Clayton, G. C. 2001, AP\&SS 275, 143

de Laverny P., Mennessier, M. O., Mignard, F., Mattei, J. A. 1998, A\&A 330, 169

Edmonds, P. D., Gilliland, R. L. 1996, ApJ 464, L157

Feast, M. W. 1986, in Hydrogen Deficient Stars and Related Objects, ed. K. Hunger (Dordrecht, Reidel), p. 151

Fekel, F. C., Henry, G. W., Henry, S. M. 2000, in Garcia Lopez, R. J., Rebolo, R., Zapatero Osorio, M. R. (eds.), 11th Cambridge Workshop on Cool

Stars, Stellar Systems and the Sun, ASP Conf. Ser. 223, 925

Freytag, B., Holweger, H., Steffen, M., Ludwig, H.G. 1997, in Paresce, F. (ed.),

Science with the VLT interferometer", ESO Astrophys.Symp., Springer, p. 316

Gillett, F. C., Backman, D. E., Beichman, C., Neugebauer, G. 1986, ApJ 310, 842 Höfner, S. 1999, A\&A 346, L9

Iben, I., Tutukov, A. V. \& Yungelson, L. R. 1996, ApJ 456, 750

Jorissen, A., Mowlavi, N., Sterken, C., Manfroid, J. 1997, A\&A 324, 578 
Kerschbaum, F., Lebzelter, T., Lazaro, C. 2001, A\&A 375, 527

Koen, C., Laney, D. 2000, MNRAS 311, 636

Lawson, W. A. \& Cottrell, P. L. 1997, MNRAS 285, 266

Lebzelter, T. 1999a, A\&A 346, 537

Lebzelter, T. 1999b, A\&A 351, 644

Lebzelter, T., Kiss, L. L., Hinkle, K. H. 2000, A\&A 361, 167

Maffei, P., Tosti, G. 1995, AJ 109, 2652

Pandey, G., Kameswara Rao, N., Lambert, D. L., et al. 2001, MNRAS 324, 937

Percy, J. R. 1997, JAAVSO 25, 93

Percy, J. R., Wilson, J. B., Henry, G. W. 2001, PASP 113, 983

Saio, H. 1995, MNRAS 277, 1393

Saio, H. \& Jeffery, C. S. 2000, MNRAS 313, 671

Schwarzschild, M. 1975, ApJ 195, 137

Walker, H. J. 1986, in Hydrogen Deficient Stars and Related Objects, ed. K. Hunger (Dordrecht, Reidel), p. 407

Walker, H. J. 1994, CCP7 Newslett. 21, 40

Weiss, A., Fried, R., Olson, C. E. 1996, A\&AS 116, 31

Woitke, P., Goeres, A. \& Sedlmayr, E. 1996, A\&A 313, 217 